\title{
Efficient phase sequence generation for SLM scheme without side information
}

\author{
Hyunju Kim ${ }^{1}$, Eonpyo Hong ${ }^{1}$, Changjun Ahn' ${ }^{2}$, \\ and Dongsoo Har ${ }^{1 \mathrm{a})}$ \\ ${ }^{1}$ Gwangju Institute of Science and Technology (GIST), \\ 261 Cheomdan-gwagiro, Buk-gu Gwangju 500-712, Republic of Korea \\ ${ }^{2}$ Chiba University, \\ 1-33 Yayoi-cho, Inage-ku, Chiba, 263-8522, Japan \\ a)hardon@gist.ac.kr
}

\begin{abstract}
Selected mapping (SLM) scheme without side information (SI) using specially designed phase symbols to reduce peak-to-average power ratio (PAPR) was recently published. The PAPR reduction performance was achieved at the cost of degraded bit error rate (BER) performance. This paper will present a new pilot phase symbol generation improving the PAPR reduction performance without BER performance deterioration.
\end{abstract}

Keywords: OFDM, PAPR, side information, SLM

Classification: Wireless communication hardware

\section{References}

[1] T. Jiang and Y. Wu, "An overview: peak-to-average power ratio reduction techniques for OFDM signals," IEEE Trans. Broadcast., vol. 54, no. 2, pp. 257-268, June 2008.

[2] M. Ohta, A. Iwase, and K. Yamashita, "Improvement of the error characteristics of N-continuous OFDM system by SLM," IEICE Electron. Express, vol. 7, no. 18, pp. 1354-1358, Sept. 2010.

[3] S. Y. L. Goff, S. S. Al-Samahi, B. K. Khoo, C. C. Tsimenidis, and B. S. Sharif, "Selected mapping without side information for PAPR reduction in OFDM," IEEE Trans. Wireless Commun., vol. 8, no. 7, pp. 3320-3325, July 2009.

[4] E. Hong, S. Min, and D. Har, "SLM-based OFDM system without side information for data recovery," Electron. Lett., vol. 46, no. 3, pp. 271-272, Feb. 2010.

[5] "Digital land mobile radio communication. Luxembourg: Final Report" (Commission of the European Communities, Final Report Office for Official Publications of the European Communities, Luxembourg, COST 207: Digital Land Mobile Radio Communications, 1989).

[6] P. Cheng, Y. Xiao, L. Dan, and S. Li, "Improved SLM for PAPR reduction in OFDM system," Proc. IEEE 18th Int. Symposium on Personal, Indoor and Mobile Radio Commun. (PIMRC), Greece, 2007.

[7] "Digital video broadcasting; frame structure, channel coding and modulation for a second generation digital terrestrial television broadcasting system," Tech. Rep. ETSI EN 302 755, V1.1.1, 2009. 


\section{Introduction}

To reduce high peak-to-average power ratio (PAPR) of orthogonal frequency division multiplexing (OFDM) systems, a variety of schemes have appeared in the literature $[1,2,3,4]$. Among these schemes, the selected mapping (SLM) scheme is considered an attractive solution because of its significant PAPR reduction performance without signal distortion. However, for recovery of the original OFDM signal at the receiver, side information (SI), e.g., index of the selected phase sequence, is also transmitted, incurring system complexity increment and data throughput loss.

To avoid such problems, several SLM schemes without SI have been published $[3,4]$. In [3], the disparity in average energy between groups of data symbols is used to recover the SI. The set of phase sequences in [4] to recover original OFDM signal in a scattered pilot pattern enables data decoding without SI. However, the PAPR reduction performance is achieved at the expense of degraded BER performance. In this paper, an efficient pilot phase symbol generation method is proposed to improve PAPR reduction performance without deteriorated BER performance.

\section{Previous scheme [4] for phase sequence generation}

The OFDM signal $x(n)$ is obtained by taking an inverse fast Fourier transform of frequency domain OFDM sequence $X(k)$ as following

$$
x(n)=\frac{1}{\sqrt{N}} \sum_{k=0}^{N_{V}-1} X(k) e^{j 2 \pi k n / N}, \quad 0 \leq n, k \leq N-1,
$$

where $N_{V}$ is the number of valid subcarriers excluding null subcarriers and $X(k)$ is modulated by phase shift keying (PSK) or quadrature amplitude modulation (QAM). The PAPR is defined as

$$
\mathrm{PAPR}=\frac{\max _{0 \leq n \leq N-1}|x(n)|^{2}}{E\left[|x(n)|^{2}\right]} .
$$

The frequency domain OFDM sequence $X(k)$ for $0 \leq k \leq N_{V}-1$ consists of $N_{p}$ pilot symbols $X(m L)$ for $0 \leq m \leq N_{p}-1$ and $N_{d}$ data symbols $X(m L+l)$ for $1 \leq l \leq L-1$, where $L=$ pilot symbol spacing, $m=$ quotient of $k / L$, and $l=$ remainder of $k / L$. The $u$-th phase sequence $P_{u}(k)$ in [4] also consists of $N_{p}$ pilot phase symbols $P_{u}(m L)$ and $N_{d}$ data phase symbols $P_{u}(m L+l)$, following the same classification rule. The pilot phase symbol $P_{u}(m L)$ for $U$ phase sequences is generated by

$$
P_{u}(m L)= \begin{cases}B_{u}(m), & m=0 \\ P_{u}((m-1) L) \cdot e^{j \frac{\pi}{R} B_{u}(m)}, & m \geq 1\end{cases}
$$

where $B_{u}(m) \in\{+1,-1\}$ is an independent and identically distributed equally probable random sequence and $R$ is the phase parameter. The data phase symbol $P_{u}(m L+l)$ is then obtained by the linear interpolation of adjacent pilot phase symbols $P_{u}(m L)$ and $P_{u}((m+1) L)$ as following

$$
P_{u}(m L+l)=\frac{L-l}{L} P_{u}(m L)+\frac{l}{L} P_{u}((m+1) L), \quad 1 \leq l \leq L-1 .
$$


Because of interpolated data phase symbol and smooth channel variation between pilot symbols, pilot-assisted channel estimation and further data decoding are possible without SI.

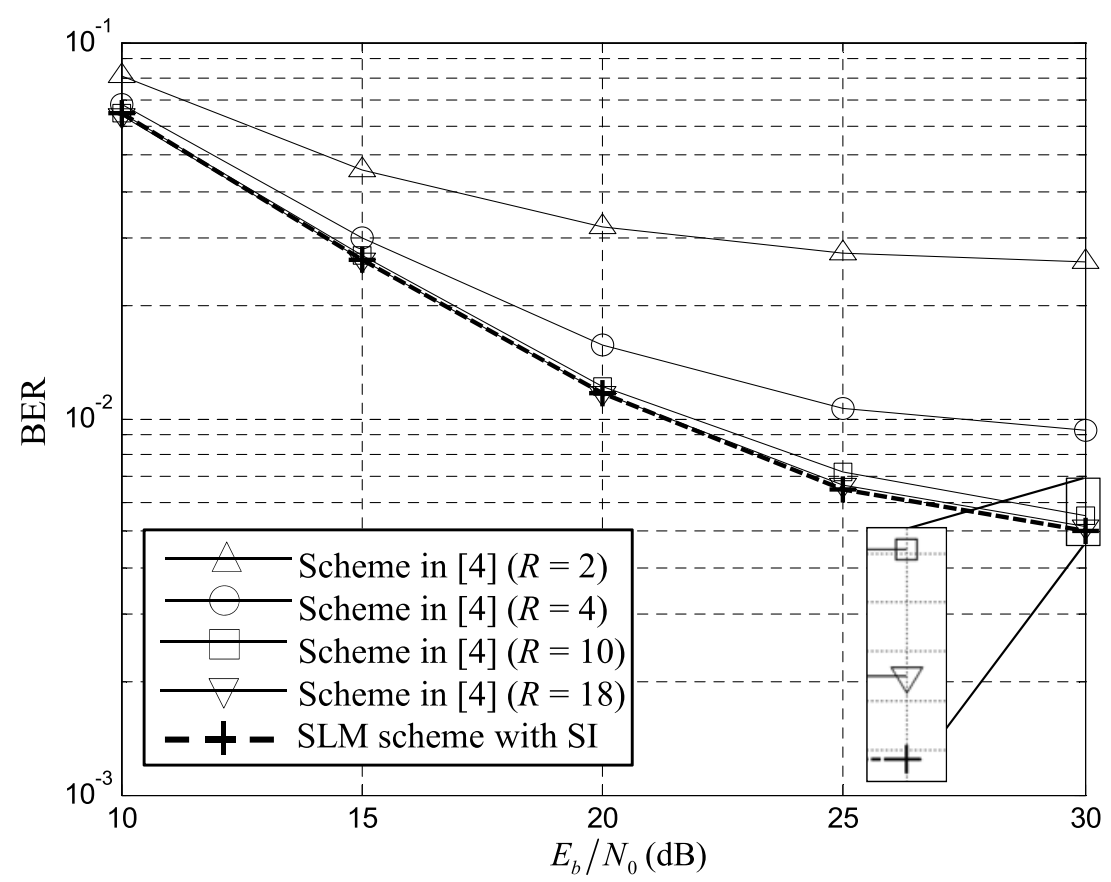

Fig. 1. Comparison of BER performances in TU-6 channel between the scheme in [4] and the SLM scheme with SI. Phase parameter $R$ varies from 2 to 18.

Fig. 1 shows BER performance according to $R$ parameter with 6-tap typical urban (TU-6) channel [5] when data symbols are modulated by 16QAM. It is conceived that the impact of small $R$ on BER performance deterioration is more significant in urban channels with more severe fading, in comparison with rural channels used for simulations in [4]. It is seen in Fig. 1 that the BER performance with small $R=2,4$ is significantly degraded compared with that of the SLM scheme with SI, but the BER performance with large $R$ such as $R=10,18$ is almost the same as that of the SLM scheme with SI.

\section{Proposed scheme}

In order to improve the PAPR reduction performance with large $R$, a new set of pilot phase sequences is proposed, which exploits the freedom in choosing available phases in the pilot symbols. The proposed pilot phase sequence is given as

$$
P_{u}(m L)=P_{u}((q G+g) L)=\left\{\begin{array}{lr}
B_{u}(0), & (q=g=0) \\
P_{u}(q G L) \cdot e^{j \frac{\pi}{R} g B_{u}(q+1)}, \quad(q \geq 0, & 1 \leq g \leq G-1) \\
P_{u}((q-1) G L) \cdot e^{j \frac{\pi}{R} G B_{u}(q)}, \quad(q>0, g=0)
\end{array}\right.
$$


where the grouping parameter $G$ for $G \geq 1$ is the number of pilot phase symbols taking the same directional phase increments, and $q, g$ are quotient, remainder of $m / G$, respectively. When $G=1, P_{u}(m L)$ in (5) is identical with that in (3). Upper limit of $G$ value is the number of pilot symbols in an OFDM symbol, though $G$ values for $G \geq R$ turned out to be bad choices for PAPR reduction in this work. In $(5), B_{u}(0)$ is randomly generated for initialization and $B_{u}(q)$ for $q \geq 1$ is repetitively used for every $G$ pilot phase symbols. Data phase symbols of the proposed scheme are also obtained from an interpolation of adjacent pilot phase symbols, as shown in (4).

For evaluation of the phase sequence generated by the proposed scheme, the variance of correlation ( $\mathrm{VC}$ ) of [6] is used as a criterion. The $\mathrm{VC}$ is expressed as

$$
\mathrm{VC}=\left(\sum_{0 \leq v<u \leq U-1} \operatorname{Var}\left\{\left|R_{u v}(\tau)\right|^{2}\right\}\right) /\left(\begin{array}{l}
U \\
2
\end{array}\right)
$$

where $\left(\begin{array}{l}U \\ 2\end{array}\right), R_{u v}(\tau), \operatorname{Var}\left\{\left|R_{u v}(\tau)\right|^{2}\right\}$ are respectively obtained as

$$
\begin{gathered}
\left(\begin{array}{c}
U \\
2
\end{array}\right)=\frac{U(U-1)}{2}, \\
R_{u v}(\tau)=\frac{1}{N} \sum_{k=0}^{N_{V}-1} P_{u}(k)\left(P_{v}(k)\right)^{*} e^{j \frac{2 \pi k \tau}{N}}, \quad 0 \leq \tau \leq N-1, \\
\operatorname{Var}\left\{\left|R_{u v}(\tau)\right|^{2}\right\}=\frac{1}{N} \sum_{\tau=0}^{N-1}\left(\left|R_{u v}(\tau)\right|^{2}-E\left\{\left|R_{u v}(\tau)\right|^{2}\right\}\right)^{2},
\end{gathered}
$$

where $E\left\{\left|R_{u v}(\tau)\right|^{2}\right\}$ is given by

$$
E\left\{\left|R_{u v}(\tau)\right|^{2}\right\}=\frac{1}{N} \sum_{\tau=0}^{N-1}\left|R_{u v}(\tau)\right|^{2} .
$$

Low VC value of candidate phase sequences means low cross-correlation between all pairs of them and enables good PAPR reduction performance. In Section 4, the VC values of the proposed scheme with various $G$ values are compared with that of the scheme in [4] and the SLM scheme. It is found that $G=R / 2$ among other $G$ values used for simulations leads to the lowest $\mathrm{VC}$ value, though $\mathrm{VC}$ values for other $G$ are not presented here.

The BER performances of the scheme in [4] and the proposed scheme are identical for common phase parameter $R$ values, as deduced from (5) for pilot phase symbols and (4) for data phase symbols. Also, with large $R$ values $(>10)$, the BER performances of these two schemes are effectively the same as that of the SLM scheme (see Fig. 1).

\section{Simulation results}

Simulation parameters following the DVB-T2 standard [7] are $N=2048$, $N_{V}=1705, L=12, N_{p}=143$, OFDM signal duration $=224 \mu s$, and modulation type $=$ BPSK for pilot symbols and 16-QAM for data symbols. The 
PAPR reduction performances of the scheme in [4] and the proposed scheme for $R=2,4$ and $G=R / 2$ are very close to that of the SLM scheme, although not presented here. For comparisons of PAPR reduction performance, simulation results corresponding to large $R$ values are shown here. In this paper, pilot symbol power and data symbol power are set to be equal. Over-sampling rate is set to 4 .

The VC values of the proposed scheme, the scheme in [4], and the SLM scheme are compared in Table I. Since only the pilot phase sequence $P_{u}(m L)$ is randomly generated for the proposed scheme and the scheme in [4], VC value is evaluated with the pilot phase sequence. Fig. 2 shows PAPR reduction performances of the proposed scheme, the scheme in [4], and the SLM scheme with SI when $U=4,16$. As seen in Fig. 2, the scheme in [4] leads to worse PAPR reduction performance, compared to that of the proposed scheme. The proposed scheme with $R=18$ and $G=9$ achieves $0.62 \mathrm{~dB}$ $(=0.76 \mathrm{~dB}-0.14 \mathrm{~dB})$ gain when $U=4$ and $0.60 \mathrm{~dB}(=0.76 \mathrm{~dB}-0.16 \mathrm{~dB})$ gain for $U=16$ over the scheme in [4]. As compared to the SLM scheme with SI, PAPR reduction performance of the proposed scheme is very close

Table I. VC of the proposed scheme in comparison with the scheme in [4] and the SLM scheme.

\begin{tabular}{l|c|c|c|c}
\hline & Scheme in [4] $(R=18)$ & SLM scheme & \multicolumn{2}{|c}{ Proposed scheme $(R=18)$} \\
\hline \multirow{2}{*}{$U=4,16$} & \multirow{3}{*}{$2.04 \times 10^{-3}$} & \multirow{2}{*}{$4.89 \times 10^{-5}$} & $G=2$ & $1.30 \times 10^{-3}$ \\
\cline { 3 - 4 } & & & $G=9$ & $6.67 \times 10^{-4}$ \\
\cline { 3 - 4 } & & & $G=16$ & $8.19 \times 10^{-4}$ \\
\hline
\end{tabular}

* $V C$ is variance of correlation.

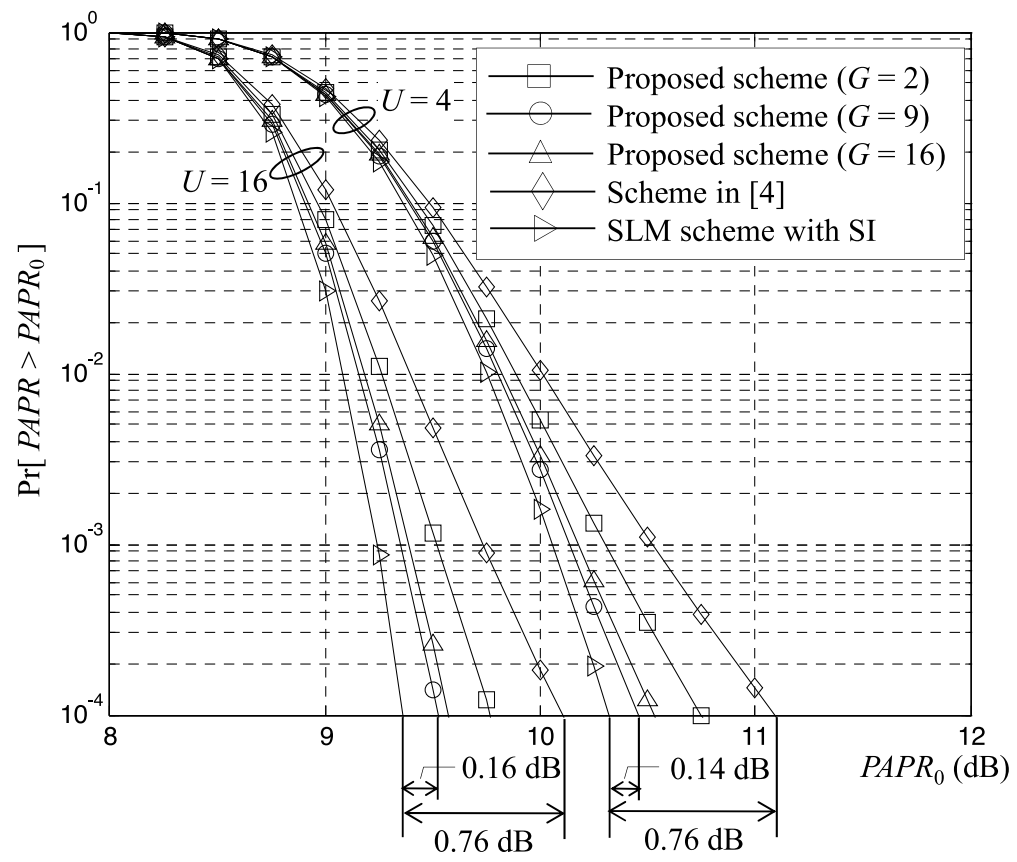

Fig. 2. PAPR reduction performances of the proposed scheme, the scheme in [4], and the SLM scheme with SI when $R=18$. For the proposed scheme $G$ values other than $G=9$ are used for comparisons. 
when $R=18$. In Fig. 2, PAPR reduction performances corresponding to various $G$ values for $R=18$ are presented. It is found that the $G=R / 2=9$ provides the best results, as expected, and the PAPR reduction performance of the proposed scheme in Fig. 2 is better than that of the scheme in [4] with all the $G$ values used for simulations.

\section{Conclusions}

In this paper, a new pilot phase symbol generation technique enabling data transmission without SI is discussed for SLM-based schemes. The aim of the proposed scheme is to achieve PAPR reduction performance gain over the scheme in [4] while maintaining low BER when phase parameter $R$ is large. Simulation results show that the proposed scheme achieves a significant improvement of PAPR reduction performance over the scheme in [4] even for large $R$ values with no degradation in BER performance. In comparison with the SLM scheme with SI, the PAPR reduction performance of the proposed scheme for practical OFDM system parameter values is very close to that of the SLM scheme. Considering the cost of transmitting SI, the proposed scheme is useful from practical point of view.

\section{Acknowledgments}

This work was supported in part by the Center for Distributed Sensor Networks at the Gwangju Institute of Science and Technology (GIST). 